\title{
Zeit der Scharlatane - die Selbstzerstörung der SPD
}

\section{Weder Ideen- noch Sozialgeschichte - die Ökonomie ist es}

Zusammenfassung: Die PROKLA 196 diskutierte in ihrem Schwerpunkt die Krise der Sozialdemokratie. Ingo Schmidt sieht in seiner ideengeschichtlichen Skizze einen von der Anziehungskraft wichtiger ökonomischer Denker geprägten Prozess der Veränderung der SPD. Manfred Wannöffel konstatiert eine schwindende Macht der Arbeiterbewegung, die soziale Basis der SPD. Beide Diagnosen nehmen die SPD aus der Verantwortung, zeichnen sie eher als Getriebene. Die These der vorliegenden Replik lautet hingegen, dass der Niedergang der SPD vielmehr das Resultat von selbstzerstörerischen Handlungen ihrer politischen Führungen ist, einem Defizit an einer theoretisch informierten, eigenständigen Wirtschaftspolitik.

Schlagwörter: Sozialdemokratie, Dritter Weg, Wirtschaftspolitik, Geldpolitik, Keynesianismus, Neoklassik, Neoliberalismus, Hartz IV

\section{Time of the charlatans - the self-destruction of the SPD}

Neither the history of ideas nor social history: it is the economy

Abstract: PROKLA 196 discussed the crisis of social democracy. Ingo Schmidt sees in his sketch of the history of ideas a process of change in the SPD that has been shaped by the attraction of important economic thinkers. Manfred Wannöffel notes a dwindling power of the labour movement, the social base of the SPD. Both diagnoses absolve the SPD of its responsibility, painting it rather as a party driven to this position. The thesis of the replica, on the other hand, is that the decline of the SPD is rather the result of self-destructive actions of its political leaders, and their deficit in terms of a theoretically-informed independent economic policy.

Keywords: Social democracy, third way, economic policy, monetary policy, Keynesianism, neoclassical economics, neoliberalism, Hartz IV

* Michael Wendl ist Soziologe, Mitherausgeber der Zeitschrift Sozialismus und hat von 1980 bis 2016 für die Gewerkschaften ÖTV und ver.di gearbeitet. 
D ie Beiträge von Ingo Schmidt und Manfred Wannöffel zur Krise der deutschen Sozialdemokratie in PROKLA 196 markieren einen offenen Widerspruch. Wannöffel betont in seiner Analyse den Wandel in den Nutzungsformen der Arbeitskraft seit den 1970er Jahren und sieht in diesen Veränderungsprozessen wichtige Gründe für die Erosion sozialdemokratischer Machressourcen. Schmidt sieht in seiner ideengeschichtlichen Skizze einen von der Anziehungskraft wichtiger ökonomischer Denker geprägten Prozess der Veränderung der SPD. Wannöffels Hinweis auf den Wandel in den Nutzungsformen der Lohnarbeit und auf die damit verbunden schwindende Macht der Arbeiterbewegung in diesem Zeitraum ist richtig. Das führt aber dazu, dass die SPD eher als Getriebene oder als passiver Faktor in sozio-ökonomischen Prozessen gesehen wird. Das erfasst nur einen Aspekt der historischen Entwicklung und blendet die aktive Rolle weitgehend aus, die die SPD in ihrer Funktion als Regierungspartei gespielt hat. Meine These lautet hingegen, dass die Erosion der sozialen Basis der SPD vielmehr das Resultat von selbstzerstörerischen Handlungen ihrer politischen Führungen ist. Schmidts Verweis auf Marx, Keynes und Hayek als ideologische Treiber der SPD ist nicht nur idealistisch, weil sie die Entwicklungen von Politik und Parteien als Folge der Theorien großer Denker missversteht. Auch in der Auswahl der Leitfiguren liegt er falsch. Sicher spielte in der frühen SPD der Marxismus in der durch Engels und Kautsky popularisierten Fassung für die Parteiintellektuellen eine Rolle, bestimmend für den Aufstieg dieser SPD wa- ren aber die Arbeits- und Lebensbedingungen der arbeitenden Klassen. Falsch ist die These, dass Keynes' Theorie die SPD der Jahrzehnte nach 1950 geprägt hatte. Sicher war der Reformismus des Godesberger Programms anschlussfähig an das standardkeynesianische Konzept der antizyklischen Globalsteuerung. Dieses stammt aber nicht von Keynes selbst, sondern basiert auf dem Versuch, Keynes' Makroökonomie in die neoklassische Gleichgewichtstheorie zu integrieren - die sogenannte neoklassische Synthese. Das Konzept hatte den in Deutschland herrschenden Ordoliberalismus kurzzeitig verdrängt, aber seine Rezeption blieb auf die Wirtschaftspolitiker und die damals noch standardkeynesianisch ausgebildeten Ökonomen in Partei und in den Gewerkschaften des DGB beschränkt. Völlig verfehlt aber ist die Annahme, die späte SPD sei durch die Sozialphilosophie oder gar die monetäre Krisentheorie von Friedrich August von Hayek beeinflusst worden. Hayek war wegen seiner politischen Nähe zu autoritären Regierungen wie etwa in Chile oder seiner Beratertätigkeit für die radikalneoliberale Margaret Thatcher eher verdächtig. Seine ökonomischen Lehren waren weitgehend unbekannt.

Wenn wir die frühen Auseinandersetzungen zwischen Schröder und Lafontaine betrachten, hat es eine wirtschaftstheoretisch begründete Konzeption der SPD nicht gegeben (Wendl 1997). Erst nach dem Ausscheiden von Lafontaine entwickelte sich eine erkennbare Linie sozialdemokratischer Wirtschaftspolitik. Sie war zunächst gekennzeichnet durch hohe Steuersenkungen für die Unternehmen und die Senkung des 
Rentenniveaus. Diese Initiativen wurden damit begründet, dass der Sozialstaat so gesichert werden könne, wenn auch in verschlankter Form.

Lafontaine hatte in seinen Monaten als Finanzminister andere Pläne. Er wollte die Steuerfreiheit für Rückstellungen in der deutschen Versicherungswirtschaft und in der Atomwirtschaft einschränken und niedrige Arbeitseinkommen entlasten. Diese beiden konträren Konzepte hatten mit entsprechenden Diskussionen in der SPD nichts zu tun. Solche Diskussionen hatte es in den Jahren vor 1998 schlicht nicht gegeben. Was nach dem Ende der sozialliberalen Koalition 1982 in der SPD vielmehr begonnen wurde zu diskutieren, war ein neues wirtschaftspolitisches Programm. Hier ging es um die Frage: Was kommt nach dem Keynesianismus?

\section{Globalisierung und Finanzialisierung}

Stärker als durch die Veränderungen in der Organisation der Lohnarbeit wurden diese Diskussionen von Fragen der ideologischen Deutung der internationalen und damit verbundenen nationalen ökonomischen Prozesse geprägt: Der Zerfall der Währungsarchitektur von Bretton Woods nach 1971, die schwere international synchronisierte Krise zwischen 1973 und 1975, die Freigabe der Wechselkurse und der Übergang der Bundesbank zur monetaristischen Geldpolitik. Unterm Strich führte das zu hoher Arbeitslosigkeit. Im Rahmen der antizyklischen Globalsteuerung konnte darauf aufgrund des Kurswechsels der Bundesbank mit öffentlichen Investitionsprogrammen nur unzurei- chend reagiert werden. Die Bundesbank hatte die Leitzinsen drastisch erhöht. Vor diesem Hintergrund kam es zu zwei Diskursen: über die Frage der Ohnmacht nationaler Wirtschaftspolitik und über den Anpassungsdruck nationaler Volkswirtschaften durch die zunehmende Internationalisierung der Produktion. Es ging erstens um die Frage, ob unter diesen neuen Bedingungen eine expansive Finanzpolitik im Rahmen der Globalsteuerung noch möglich sei. Zweitens wurde diskutiert, ob die Freigabe der Wechselkurse und die Hochzinspolitik der US-amerikanischen Zentralbank Fed unter Paul Volcker zu einem Verlust der nationalen Zinssouveränität geführt habe. In beiden miteinander eng verbundenen Diskursen wird die Debatte über das Ende des Keynesianismus geführt. Das geschah auch von Seiten linker Ökonomen (Altvater, Hübner, Stanger 1983). Von neoliberalen Ökonomen wie Hayek und Milton Friedman wurde im Rahmen der Mont-Pèlerin-Society seit den 1930er Jahren auf eine Zurückdrängung des Keynesianismus hingearbeitet (Walpen 2004). Diese begrüßten eine offene Weltwirtschaft weitgehend unregulierter Märkte. Diese Diskurse spielten für die SPD als Partei und für die Gewerkschaften jedoch zunächst kaum eine Rolle. Ihre Sicht auf den aktuellen Kapitalismus war nicht ökonomietheoretisch informiert oder gar geprägt, also weder marxistisch, keynesianisch oder gar neoklassisch. Genau das war das große Defizit, denn die SPD stellte den neuen ökonomischen Bedingungen keine neue, eigenständige Idee entgegen, wie Wirtschaftspolitik aussehen könnte - und wurde mit der Zeit durch 
die wirtschafts- und wissenschaftspolitische Dominanz der Neoklassik unterhöhlt, die zunehmend den Alltagsverstand prägte.

Vielmehr hatte sich Mitte der 1980er Jahre ein wirtschaftspolitischer Kurswechsel in der SPD und später in den Gewerkschaften angebahnt. Angestoßen hatte ihn Fritz Scharpf (1987), der in seiner Bilanz der sozialdemokratischen Krisenpolitik in Europa die Machtlosigkeit einer beschäftigungsorientierten Finanzpolitik, aber auch das Ende der nationalen Geldpolitik diagnostiziert hatte. Letzteres war falsch, weil die Bundesbank mit ihrer monetaristischen Wende und ihrer Hochzinspolitik nach 1973 das Gegenteil bewiesen hatte. Sie setzte damals ihre Zinssouveränität gezielt dazu ein, die Gewerkschaften zu disziplinieren. Doch damit erschwerte sie gleichzeitig eine beschäftigungsorientierte Finanzpolitik durch ihre restriktive Geldpolitik. Das sogenannte ISLM-Modell der neoklassischen Synthese, mit dem der Gütermarkt (InvestmentSavings) und der Kapitalmarkt (Liquidity-Money-Supply) Gleichgewichte erreichen sollen, konnte nicht mehr angewendet werden. Das galt ebenso für die Phillips-Kurve, nach der es eine gegenläufige Beziehung (Trade-Off) zwischen steigender Arbeitslosigkeit und steigender Inflation geben sollte. ${ }^{1}$ Vor diesem politischen Hintergrund blieb nur die nationale Arbeitsmarktpolitik, um Beschäftigung zu sichern und auszu-

1 Elmar Altvater hatte das Buch von Scharpf als "angebotspolitischen Keynesianismus« kritisiert, obwohl er Keynes und Hyman P. Minsky zustimmend gegen diesen »Keynesianismus« zitiert hatte (Altvater 1988). weiten, nachdem Finanz- und Geldpolitik als Steuerungsinstanzen ausfielen.

Die Analyse von Scharpf, die in der SPD und in den Gewerkschaften zunächst kaum rezipiert wurde, hatte direkten Einfluss auf die Initiative von Oskar Lafontaine, Arbeitszeitverkürzung mit einem Lohnverzicht bei höheren Arbeitsentgelten zu verbinden. Die IG Metall und die IG Druck und Papier hatten mit Arbeitskämpfen in den 1980er Jahren erste Schritte zur 35-Stunden-Woche durchsetzen können. Lafontaines Initiative scheiterte zunächst am Widerstand des linken SPD-Flügels und der großen Mehrheit der Gewerkschaften.

\section{Die fatale Rolle der Gewerkschaften}

Der tarifpolitische Kurswechsel der DGBGewerkschaften fand erst 1995 statt. Die IG Metall hatte ein erstes Angebot für ein »Bündnis für Arbeit« vorgelegt und dafür Beschäftigungszusagen der Unternehmen erwartet. Sie wäre bereit gewesen, den Verteilungsspielraum der produktivitätsorientierten Tarifpolitik nicht auszuschöpfen, um so zusätzliche Beschäftigung zu finanzieren. In der Sache war das die Übernahme der neoklassischen Lohntheorie, wie sie der Sachverständigenrat zur Begutachtung der gesamtwirtschaftlichen Entwicklung (SVR) in seinen Gutachten seit Mitte der 1970er Jahre vertreten hatte. Die Lohnzurückhaltung sollte zusätzliche Investitionen anregen. In der Theorie war das auch die Position von Scharpf, aber dieser wollte die Lohnmoderation in einen internen Umverteilungsprozess einbinden, den er "Sozialismus in einer Klasse« nannte. Hier wurde der Lohn- 
verzicht bei höheren Einkommen als Geste der Solidarität mit den Arbeitslosen gewertet. Der Kern dieses Konzepts bestand darin, niedrige Arbeitseinkommen staatlich zu subventionieren, um im Dienstleistungssektor neue Beschäftigung zu schaffen. Dahinter stand die für eine auf Exportüberschüsse getrimmte Ökonomie wenig plausible These von einer deutschen Dienstleistungslücke aufgrund geringer Lohnflexibilität (zur Kritik an diesem Narrativ: Haisken-deNew u.a. 1998). Für die zu hohen Dienstleistungslöhne wurden ein überregulierter Arbeitsmarkt und hohe Lohnnebenkosten verantwortlich gemacht.

Lafontaine hatte in den 1990er Jahren seine frühere, von Scharpf geprägte Sicht deutlich revidiert, weil er unter dem Einfluss von Heiner Flassbeck auf einen postkeynesianischen Kurs wechselte. Die »Ideologie vom Sozialismus in einer Klasse« blieb unter den Parteiintellektuellen populär. Die neue wirtschaftspolitische Position von Lafontaine hingegen hatte in der Partei nur eine schmale Basis. Lafontaine hatte es versäumt, seine neue keynesianische Sicht in der Partei bekannt zu machen (Wendl 1997). Ökonomietheoretisch klaffte in der Partei weiterhin eine Lücke. Spätestens im März 1999, als er kapitulierte, war der kurze Sommer des Keynesianismus in der SPD zu Ende. Lafontaine wäre vermutlich auch im Fall eines Nicht-Rücktritts gescheitert, weil der Konflikt nicht in der SPD, sondern letztlich im Regierungsapparat geführt wurde. Er konnte auch nicht mit der Unterstützung der Gewerkschaften rechnen. In den Industriegewerkschaften, aber auch in der ÖTV, hatte sich die Sicht durchgesetzt, dass die Arbeitskosten im "westdeutschen Hochlohnsystem « für den internationalen Wettbewerb zu hoch seien (Streeck 1999: 34). Ausschlaggebend für diese Sicht waren die gestiegenen Beiträge zur Sozialversicherung, die eine Debatte über zu hohe Lohnnebenkosten befeuerten. SPD- und Gewerkschaftsvertreter berücksichtigten dabei allerdings nicht, dass bei unterhalb des Produktivitätszuwachses steigenden gesamtwirtschaftlichen Lohnstückkosten die Lohnnebenkosten nicht zu hoch sein können, da sie in der Größe der Lohnstückkosten komplett enthalten sind. Das zeigt, dass eine postkeynesianische Position, zu deren Selbstverständnis diese Tatsache gehört, in dem politischen Feld zwischen SPD und Gewerkschaften damals kaum eine Chance hatte. Möglicherweise wäre es anders gekommen, wenn Lafontaine nach seiner Wahl zum Parteivorsitzenden eine wirtschaftspolitische Debatte in der SPD initiiert hätte. Doch er hatte wie viele Spitzenpolitiker nicht vor, mit seiner Partei zu regieren. Er meinte, dass es reicht, wenn er und seine Berater regieren. Die Mobilisierung sozialdemokratischer Machtressourcen (Wannöffel 2019: 372) war nicht vorgesehen.

\section{Steuersenkungen und Austerität}

Schröders erster Akt nach der Demission Lafontaines waren groß dimensionierte Steuersenkungen, insbesondere für Unternehmen durch die Senkung und die nachträgliche Erstattung vor 2000 bezahlter Körperschaftsteuern. Dahinter stand die simple Sicht, die Schröder auch weder im Regierungsapparat noch in der Partei weiter ökonomietheore- 
tisch begründen musste, dass niedrige Steuern internationales Kapital anziehen und damit Beschäftigungsimpulse auslösen. Die von Rot-Grün veranlassten Steuerausfälle kumulierten sich bis 2010 auf insgesamt 762,5 Milliarden Euro (Truger 2009: 33) und zwangen die Regierung zu einer restriktiven Fiskalpolitik. Das Absenken des Rentenniveaus, das Zwangssparen für eine kapitalgedeckte Zusatzversicherung und der Verzicht auf eine aktive Konjunkturpolitik ab 2002 resultierten in einem schwachen Binnenkonsum, wodurch die seit der Wiedervereinigung hohe Arbeitslosigkeit weiter anstieg.

Das hat es den Wirtschaftsliberalen leicht gemacht, weiterhin von Deutschland als »krankem Mann" Europas zu sprechen. Dieser Mythos wurde durch die Krise 2001/02 forciert, die die Zahl der registrierten Arbeitslosen auf 4,3 Millionen ansteigen ließ. Als Gründe für diese Entwicklung wurden, wie im neoklassischen Paradigma systematisch angelegt, $\mathrm{zu}$ hohe Arbeitskosten behauptet - etwa vom SVR. Das war absurd, weil zwischen 2000 und 2005 der Außenhandelsüberschuss von knapp 60 auf 159 Milliarden Euro geradezu explodiert war, ein Prozess, der auf der Lohnzurückhaltung ab 1996 basiert hatte. ${ }^{2}$ Durch die Lohnmoderation im »Bündnis für Arbeit« von 2000 bis 2005 wurde dieser Handelsmerkantilismus verstärkt. Die Wettbewerbsfähigkeit der deutschen Exportwirtschaft war nach 1995 deutlich gestiegen und war, gemessen an den Außenhandelsüber-

2 »Gesamtentwicklung des deutschen Außenhandels ab 1950« (1.11.2019), https://www. destatis.de/. schüssen und entgegen der öffentlichen Meinung in Deutschland, nie gefährdet. Das war auch im Bundeskanzleramt bekannt, welches die Wettbewerbsfähigkeit der deutschen Exportwirtschaft als "exzellent « und die Binnennachfrage als zu schwach bezeichnete (Bundeskanzleramt 2002). Daraus wurde jedoch der Schluss gezogen, dass der »Faktor Arbeit« entlastet werden müsste, damit die Menschen »mehr konsumieren« können. Mehr »Netto vom Brutto « wurde zur neuen Zauberformel. Nichts zeigt das Elend sozialdemokratischer Wirtschaftskompetenz treffender als diese Aussagen, denn: Kürzen der Staat und die Sozialversicherungen ihre Ausgaben, führt das zu einer niedrigeren Gesamtnachfrage - und verstärkt das Problem einer zu schwachen Binnennachfrage.

Die rot-grüne Wirtschaftspolitik zeitigte zwei Pointen. Einmal hat sie in einer Zeit, in der von der Ohnmacht nationaler Wirtschaftspolitik gesprochen wurde, die Macht des Nationalstaats erfolgreich zur Stärkung marktkonformer Prozesse eingesetzt. Sie hatte damit, ohne es zu wissen, im Sinne der Sozialphilosophie Hayeks gehandelt. Dieser wollte durch politische Macht die Freiheit der Märkte durchsetzen und auf dieser Ebene Demokratie ausschließen (Slobodian 2019). Insofern enthält Schmidts Hinweis auf Hayek eine stille Ironie.

\section{Die Vorbereitung der Agenda 2010}

Die zweite Pointe liegt darin, dass die finanzpolitischen und rentenpolitischen Fehlentscheidungen von RotGrün die Arbeitslosigkeit unmittelbar nach der Krise 2001/02 auf dem hohen Niveau hielten. Es folgte eine weitere 
Fehlentscheidung, die Neuregulierung des Arbeitsmarktes, die auf nach unten flexible Löhne (Hartz IV) abzielte. Die Bundesregierung hatte sich selbst zur arbeitsmarktpolitischen Radikalkur gezwungen und folgte dabei den »simplen Vorstellungen des neoklassischen Paradigmas« (Herr 2002: 523). Auch hier machte sich das Defizit geltend, dass die SPD über keine eigene theoretisch informierte wirtschaftspolitische Konzeption verfügte: So übernahm die SPD einen Teil der Vorschläge, die der Sachverständigenrat mit seinen 20 Punkten für mehr Beschäftigung im Jahresgutachten 2001/02 gemacht hatte (SVR 2001). Doch dass das auf einem Parteitag der SPD mit 80 Prozent Zustimmung akzeptiert wurde, hat nichts mit Hayeks Sozialphilosophie zu tun. Die war auch damals den Delegierten sicherlich unbekannt. Vielmehr begründen selbst linke SPD-Abgeordnete bis heute diese Entscheidungen mit der Höhe der Arbeitslosigkeit. Das entspricht dem ökonomischen Alltagsverstand, der Arbeitslosigkeit als Resultat von zu hohen Löhnen und nicht von unzureichender aggregierter Nachfrage versteht. Die politischen Akteure der SPD handelten insofern bewusst, aber ohne hinreichendes Bewusstsein über den makroökonomischen Zusammenhang, in dem sie agierten. Die Neoklassik war inzwischen zum "gesunden Menschenverstand « avanciert. Daher brauchen die Akteure ökonomische Narrative, an denen sie sich orientieren und in denen die neoklassischen Prämissen aufgehoben sind. Das sind hier zwei Glaubenssätze: Einmal die Überzeugung, dass der Standort Deutschland in einer schärfer werdenden Konkur- renz permanent bedroht ist und zum zweiten die Überzeugung, dass eine Beschäftigungspolitik, die auf expansive Finanzpolitik und expansive Geldpolitik setzt, zu Inflation führt und damit den Standort bedroht. Deshalb gab es auch keine sozialdemokratische Kritik am monetaristischen Kurs der Bundesbank, abgesehen vom Unmut Helmut Schmidts über die Geldpolitik der Bundesbank nach 1975. Bei der Verfolgung dieser Glaubenssätze ging Rot-Grün mit einem hohen Maß an sozialer Rücksichtslosigkeit vor. Das stieß in der SPD auf Kritik. Eine ernsthafte, theoretisch informierte wirtschaftspolitische Debatte folgte daraus jedoch nicht.

Die moralische Ablehnung der Hartz-Gesetze blieb folgenlos, weil diese als Erfolg sozialdemokratischer Arbeitsmarktpolitik gewertet und letztlich sogar für den Anstieg der Zahl der Beschäftigten nach 2006 und nach 2012 verantwortlich gemacht wurden. Dieser Anstieg war jedoch in erster Linie eine Folge der Belebung der internationalen Konjunktur und des steigenden Anteils der Schwellenländer an der Nachfrage nach deutschen Investitionsgütern. Dennoch wird bis heute bei der SPD das Narrativ gepflegt, dass die Höhe der Beschäftigung mit den Funktionsbedingungen des Arbeitsmarktes zusammenhängt, das heißt vor allem mit der Lohnhöhe und der Flexibilität der Beschäftigten. ${ }^{3}$

3 Das prägt nahezu die gesamte mikroökonomische Arbeitsmarktforschung, die nicht müde wird, den Rückgang der Arbeitslosigkeit den Hartz-Gesetzen zuzuschreiben. Allerdings werden zunehmend die Folgeschäden des Niedriglohnsektors auf das Lohnniveau und das Rentensystems diskutiert. 
Dieser Prozess seit 1999 war eine resignativ hingenommene Selbstzerstörung sozialdemokratischer Werte. Dass die Mehrheit der DGB-Gewerkschaften den Kurs der SPD zwischen 1999 und 2003 im „Bündnis für Arbeit« unterstützt hatte und erst nach dem März 2003 vorsichtig auf Distanz gegangen war, schwächte die ohnehin bereits schwache innerparteiliche Opposition zusätzlich. Die Gewerkschaften waren den ideologischen Diskursen der SPD und der SPD-nahen Sozialwissenschaft gefolgt, weil sie eine ähnliche Wahrnehmung der Folgen der Prozesse der Globalisierung hatten. ${ }^{4}$

\section{Ein Weg zurück zur Sozialdemokratie?}

Die Gewerkschaften konnten sich dank des Exportbooms nach der Krise 2009 tarifpolitisch wieder stabilisieren. Dass der Einbruch der Exporte nach 2009 rasch überwunden werden konnte, lag einerseits an einem kurzfristigen keynesianischen Konjunkturprogramm und der Steuerung des Arbeitsmarkts durch die Ausweitung der Kurzarbeit und durch eine flexible Handhabung der Arbeitszeitregeln in den Tarifverträgen der Industrie. Trotz dieser Erfolge zog die SPD keine ökonomischen oder wirtschaftspolitischen Schlüsse und stimmte mit den Unionsparteien 2009 für die Einführung der Schuldenbremse im

4 Der Prozess, der als Neoliberalisierung der SPD oder als Marktsozialdemokratie bezeichnet wurde, war eine Folge der Diskurse der SPD-nahen Sozialwissenschaft, die im Arbeitsmarkt den einzigen Ansatzpunkt der Beschäftigungspolitik gesehen hatte. Das war neoklassische Ideologie, der die SPD und zeitweise auch die Gewerkschaften folgten.
Grundgesetz. Die hohe Nachfrage nach deutschen Exporten auf den internationalen Märkten führte über die Stabilisierung der Beschäftigung zum spürbaren Rückgang der Arbeitslosigkeit und die Gewerkschaften gelang nach 2011 der Übergang auf den Pfad der produktivitätsorientierten Tarifpolitik. Infolge höherer Staatseinnahmen kam es auch im öffentlichen Sektor wieder zu höheren Lohnabschlüssen. Dass es rund 10 Jahre danach auch in der SPD zu einer wirtschaftspolitischen Neuorientierung kommt, liegt daran, dass die Folgen der rot-grünen Wirtschafts- und Sozialpolitik offen zu Tage treten: ein enormer Anstieg sozialer Ungleichheit, besonders zwischen 2000 und 2006, eine marode öffentliche Infrastruktur und die Zunahme offener Armut. Angesichts dieser Entwicklungen hat sich die SPD als Partei von der SPD an der Regierung distanziert. Dass Scholz nicht zum Parteivorsitzenden gewählt wurde, ist dafür ein Zeichen. Das wirtschaftspolitische Defizit der SPD ist damit jedoch noch lange nicht überwunden.

\section{Literatur}

Altvater, Elmar (1988): Nationale Wirtschaftspolitik unter Bedingungen globaler ,finanzieller Instabilitäten «. Zu Fritz Scharpfs "angebotspolitischem Keynes ianismus«. In: PROKLA 72 18(3): 121-136. DOI: https://doi.org/10.32387/prokla. v18i72.1289.

Altvater, Elmar / Hübner, Kurt / Stanger, Michael (1983): Alternative Wirtschaftspolitikjenseits des Keynesianismus. Opladen. DOI: https://doi.org/10.1007/978-3-32283981-7.

Bundeskanzleramt (2002): Aufdem Weg zu mehr Wachstum, Beschäftigung und Gerechtigkeit. Thesenpapier für die Planungsklausur am 5.12.2002. Berlin. 
Haisken-deNew, John / Horn, Gustav Adolf / Schupp, Jürgen / Wagner, Gert G. (1998): Das Dienstleistungs-Puzzle. Ein deutsch-amerikanischer Vergleich. In: DIW Wochenbericht 65(35): 625-629.

Herr, Hansjörg (2002): Arbeitsmarktreform und Beschäftigung über die ökonomietheoretischen Grundlagen der Vorschläge der Hartz-Kommission. In: PROKLA 129 32(4): 515-536. DOI: https://doi. org/10.32387/prokla.v32i129.684.

Sachverständigenrat zur Begutachtung der gesamtwirtschaftlichen Entwicklung (2001): Jahresgutachten 2001/02. Bundesdrucksache 14/7569.

Scharpf, Fritz W. (1987): Sozialdemokratische Krisenpolitik in Europa. Frankfurt/M.New York.

Slobodian, Quinn (2019): Globalisten. Das Ende der Imperien und die Geburt des Neoliberalismus. Berlin.
Streeck, Wolfgang (1999): Deutscher Kapitalismus. Gibt es ihn? Kann er überleben? In: Korporatismus in Deutschland. Frankfurt/M.: 13-40.

Truger Achim (2009): Ökonomische und soziale Kosten von Steuersenkungen. Das Beispiel der rot-grünen Steuerreform. In: PROKLA 154 39(1): 27-46. DOI: https:// doi.org/10.32387/prokla.v39i154.440.

Walpen, Bernhard (2004): Die offenen Feinde und ihre Gesellschaft. Eine hegemonietheoretische Studie zur Mont Pèlerin Society. Hamburg.

Wannöffel, Manfred (2019): Umbruch der Produktionsweise und die Krise des sozialdemokratischen Modells. In: PROKLA 196 49(3): 371-386. DOI: https://doi.org/10. 32387/prokla.v49i196.1831.

Wendl, Michael (1997): Die SPD - ratlos zwischen Angebots- und Nachfragepolitik. In: WIDERSPRUCH 17(34): 79-88.

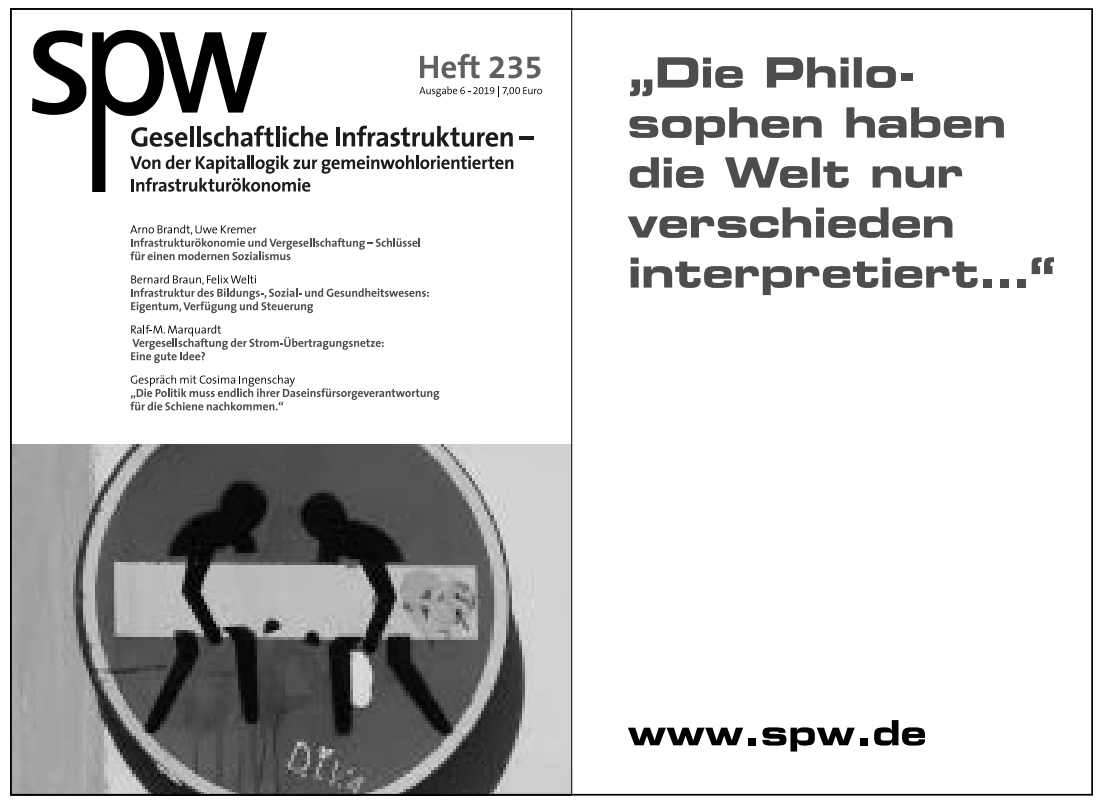




\section{WISSENSCHAFT FÜR DIE PRAXIS}

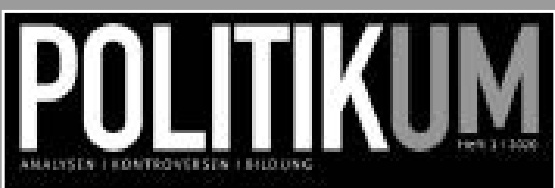

\section{EMOTIONEN}

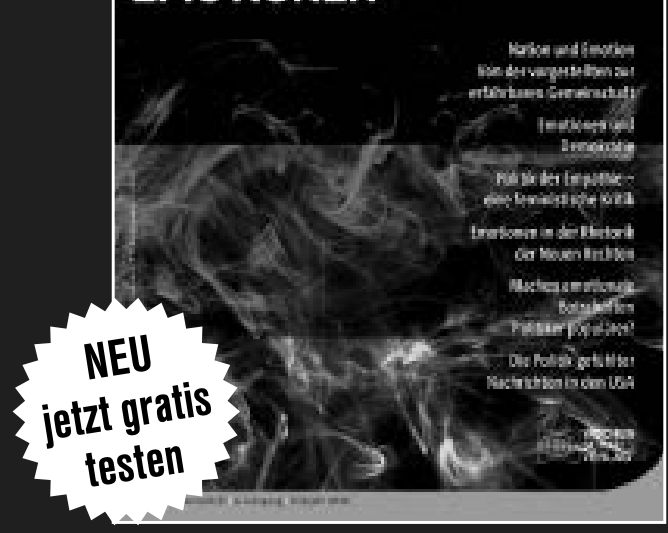

Im aktuellen Heft: Emotionen in der Politik I Auch Gefühle sind Fakten I Nation und Emotion I Emotionen und Demokratie I Politik der Empathie - eine feministische Kritik I Emotionen in der Rhetorik der Neuen Rechten I Emotionale Politiker - populäre Politiker? I Die Politik gefühlter Nachrichten in den USA

\section{FORDERN SIE JETZT IHR GRATIS-PROBEHEFT AN WWW.POLITIKUM.ORG}

